Military Technical College Kobry El-Kobba, Cairo, Egypt

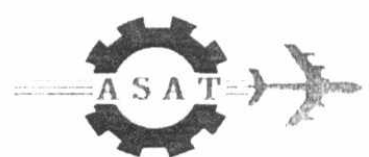

11-th International Conference on Aerospace Sciences \& Aviation Technology

\title{
OPTIMUM RECEPTION OVER IMPULSIVE NOISE CHANNELS
}

\author{
EI-barbary K. A., EL-Mahdy A. E. ", and EL shawadfy S. A."
}

\section{ABSTRACT}

The paper presents an optimum receiver for digitally modulated signals in additive class-A impulsive noise. The receiver is based on maximum likelihood (ML) approach. The performance of the receiver is derived theoretically. The performance is measured by the bit error probability. Computer simulations are illustrated to validate the theoretical developments. It is shown that the performance of the developed receiver is sensitive to the impulsive index of the noise. Decreasing the value of the impulsive index degrades the performance of the receiver and reduces the receiver resistivity against frequency offset.

KEY WORDS: non-Gaussian noise, optimum reception.

\footnotetext{
- Egyptian Armed Forces
} 


\section{Introduction}

In most of digital communication systems, the additive white Gaussian noise (AWGN) channel model is widely applied. However, the assumption of white Gaussian noise alone is sometimes not true. In several situations, the noise accompanied with the signal is non-Gaussian [1]. For example, the communication channels usually exhibit impulsive characteristics, i.e., long bursts due to noise impulses of large amplitude relative to the root mean square noise [2]. Also, the noise generated by a variety of natural and man-made electromagnetic sources exhibits impulsive characteristics [2], [3]. Non-Gaussian impulsive noise is known to be one of the major sources of errors in digital transmission systems. Therefore, a more realistic noise model might be an additive mixture of the Gaussian thermal noise and a non-Gaussian impulsive noise. One of the models that has been proposed to meet these requirements, is the general model derived by Middleton. According to the relation between the durations of the noise impulses and the spectral bandwidth of the receiver, Middleton derived three general classes of the impulsive noise: class-A, B, and C [4], [5]. Class-A model represents interference arising from sources whose emission spectra are equal to or narrower than the receiver bandwidth. Class-B model represents interference arising from sources whose emission spectra are broader than the band pass of the receiver while class- $C$ interference is composed of the sum of class- $A$ and class-B components. In this paper, we use class-A impulsive noise model because it is known to fit closely a variety of non-Gaussian noises and also it is analytically tractable model of Gaussian/non-Gaussian noises.

In this paper, the problem of optimum reception of digitally modulated signals contaminated with class-A impulsive noise is addressed. The receiver is based on maximum likelihood (ML) approach. The performance of the receiver is derived theoretically and validated by simulation for a QPSK signal as a case study. The paper is organized as follows. In section II, the class-A impulsive noise model are briefly reviewed. In section III, the optimal receiver structure for class-A impulsive noise is derived. In section IV, the theoretical performance of the receiver is evaluated for a QPSK signal. The performance is measured in terms of the bit error probability. In section $\mathrm{V}$, the simulation experiments are presented to validate the theoretical performance of the receiver. Finally, conclusions are presented in section VI.

\section{Class-A Impulsive Noise Models}

Class-A impulsive noise model of Middleton is a generalized model of the Gaussian noise combined with a non-Gaussian impulsive noise. Further details of this model are found in [5], [6]. In this model, a frequency component of the impulsive noise is constrained within the bandwidth of the receiver. The normalized class-A impulsive noise, for complex channels, has a density function $f(n)$, given by [4], [7, pp. 86]:

$$
f(n)=\sum_{m=0}^{\infty} \frac{e^{-A} A^{m}}{m !\left(2 \pi \sigma_{m}^{2}\right)} \exp \left(-\frac{|n|^{2}}{2 \sigma_{m}^{2}}\right)
$$


where the parameter $A$ is called the impulsive index: it is the product of the received average number of impulses per unit time and the duration of an impulse. This parameter defines the impulsiveness of the noise. For small $A$, the noise becomes more impulsive, that is $f(n)$ exhibits large impulsive "tails" and for larger A, the statistical characteristics of the class-A impulsive noise approach those of Gaussian noise. The variances $\sigma_{m}^{2}$ are related to the physical parameters and are given by:

$$
\sigma_{m}^{2}=\sigma_{n}^{2} \frac{(m / A)+\Gamma}{1+\Gamma} ; \quad m=0,1,2, \ldots
$$

where the parameter $\sigma_{n}^{2}$ defines the mean variance of the class-A impulsive noise. The model of the class-A noise combines the presence of an additive man-made noise component with variance $\sigma_{l}^{2}$ and a white Gaussian noise component with variance $\sigma_{G}^{2}$. The parameter $\Gamma$ in (2) is the ratio of the mean power of the Gaussian noise component to the non-Gaussian impulsive noise component. The white Gaussian noise component is presented in the class-A noise model to describe the influence of thermal noise which is naturally present in the real physical receiver. Note that, $f(n)$ consists of an infinite weighted sum of zero mean Gaussian densities with decreasing weights and increasing variances.

\section{Mathematical Formulation of the Optimum Receiver}

In this section, the optimal receiver structure under class-A impulsive noise is derived. The receiver is based on $\mathrm{ML}$ approach. The general signal detection problem is considered. One out of $M$ signals is transmitted and contaminated by class-A non-Gaussian noise. This model is denoted by $s_{i}(k) ; k=1,2, \ldots, K$, the index $i$ is an integer so that $1 \leq i \leq M, K$ is the number of samples per symbol, and $M$ is the number of possible signals. The problem can be formulated as a multiple hypotheses testing problem, that is, it is assumed that the received observation contains the signal $s_{i}(k)$ under the hypothesis $H_{i}$. Then, under hypothesis $H_{i}$, the discrete time received signal is given by

$$
H_{i}: r(k)=s_{i}(k)+n(k) ; \quad k=1,2, \ldots, K
$$

where $n(k)$ are independent and identically distributed (i.i.d) complex valued zero mean white impulsive noise samples. It is required to derive an optimal receiver, in the sense of minimization the probability of error, to determine which signal, $s_{i}(k)$, has been sent from the $M$ signals. The ML receiver chooses the hypothesis $H_{i}$ with the largest likelihood function (LF). The LF of the received signal $s_{i}(k)$ is obtained using (1) and (3), and can be expressed as: 


$$
f\left(r(k) / s_{i}(k)\right)=\sum_{m=0}^{\infty} \frac{e^{-A} A^{m}}{m ! 2 \pi \sigma_{m}^{2}} \exp \left\{\frac{-1}{2 \sigma_{m}^{2}}\left|r(k)-s_{i}(k)\right|^{2}\right\}
$$

This likelihood function (LF) can not be evaluated because of the infinite sum over $m$. The infinite sum in (4) can be approximated by the maximum value of its first three terms under the condition that the impulsive index $A$ is sufficiently small $(A<1)$ [3]. This approximation is found to be sufficient to give excellent approximation to the class-A impulsive noise probability density function [3]. Hence, (4) can be written as:

$$
f\left(r(k) / s_{i}(k)\right)=\max _{m=0,1,2}\left\{\frac{e^{-A} A^{m}}{m ! 2 \pi \sigma_{m}^{2}} \exp \left\{\frac{-1}{2 \sigma_{m}^{2}}\left|r(k)-s_{i}(k)\right|^{2}\right\}\right\}
$$

The evaluation of (5) is performed as follows [3]:

$$
f\left(r(k) / s_{i}(k)\right)= \begin{cases}\frac{e^{-A}}{2 \pi \sigma_{0}^{2}} \exp \left\{\frac{-1}{2 \sigma_{0}^{2}}\left|r(k)-s_{i}(k)\right|^{2}\right\} & \text { if } 0 \leq\left|r(k)-s_{i}(k)\right|<a \\ \frac{e^{-A} A}{2 \pi \sigma_{1}^{2}} \exp \left\{\frac{-1}{2 \sigma_{1}^{2}}\left|r(k)-s_{i}(k)\right|^{2}\right\} & \text { if } a \leq\left|r(k)-s_{i}(k)\right|<b \\ \frac{e^{-A} A^{2}}{4 \pi \sigma_{2}^{2}} \exp \left\{\frac{-1}{2 \sigma_{2}^{2}}\left|r(k)-s_{i}(k)\right|^{2}\right\} & \text { if } b \leq\left|r(k)-s_{i}(k)\right|\end{cases}
$$

where $a$ and $b(>0)$ are given by:

$$
\begin{aligned}
& a=\sqrt{\frac{2 \sigma_{0}^{2} \sigma_{1}^{2}}{\sigma_{0}^{2}-\sigma_{1}^{2}} \ln \left(\frac{\sigma_{0}^{2}}{\sigma_{1}^{2}} A\right)} \\
& b=\sqrt{\frac{2 \sigma_{1}^{2} \sigma_{2}^{2}}{\sigma_{1}^{2}-\sigma_{2}^{2}} \ln \left(\frac{\sigma_{1}^{2}}{2 \sigma_{2}^{2}} A\right)}
\end{aligned}
$$

For $K$ independent and identically distributed (i.i.d) samples, the probability density function (pdf) of the received signal, $\mathrm{r}=[r(1), r(2), \ldots, r(K)]$, given the transmitted one, $\mathbf{s}_{i}=\left[s_{i}(1), s_{2}(2), \ldots, s_{i}(K)\right]$, can be written as:

$$
f\left(\mathbf{r} / \mathbf{s}_{i}\right)=\prod_{k=1}^{K} f\left(r(k) / s_{i}(k)\right)
$$


Then, the log likelihood function (LLF) can be expressed as:

$$
\Delta_{i}\left(\mathbf{r} / \mathbf{s}_{i}\right)=\sum_{k=1}^{K} \max _{m=0,1,2}\left\{\ln \left(\frac{e^{-A} A^{m}}{m ! 2 \pi \sigma_{m}^{2}}\right)-\frac{1}{2 \sigma_{m}^{2}}\left|r(k)-s_{i}(k)\right|^{2}\right\}
$$

The ML receiver evaluates (9) for all hypothesis $H_{i}, i=1,2, \ldots, M$ and selects the hypothesis that maximizes it. The structure of this receiver is shown in Fig. (1).

\section{Performance Evaluation of the Developed Receiver}

In this section, the theoretical performance of the receiver is evaluated when the transmitted signal is QPSK. The performance is measured in terms of the probability of error as a function of SNR and the noise parameters. The QPSK signal constitutes four hypothesis corresponding to the signals $s_{0}(t), s_{1}(t), s_{2}(t)$, and $s_{3}(t)$ that represent the transmitted symbols. This is illustrated in the constellation diagram shown in Fig.2. To derive the probability of bit error, the noise is represented by its in-phase component $x(t)$ and quadrature component $y(t)$. The joint probability density function (pdf) of the in-phase component and quadrature component is given by [3]

$$
f_{x, y}(x, y)=\sum_{m=0}^{\infty} \frac{e^{-A} A^{m}}{m !\left(2 \pi \sigma_{m}^{2}\right)} \exp \left(-\frac{|x|^{2}+|y|^{2}}{2 \sigma_{m}^{2}}\right)
$$

Integrating (10) with respect to $x$ and $y$, we can obtain the pdf's of each component as:

$$
\begin{aligned}
& f_{x}(x)=\sum_{m=0}^{\infty} \frac{e^{-A} A^{m}}{m ! \sqrt{2 \pi \sigma_{m}^{2}}} \exp \left(-\frac{|x|^{2}}{2 \sigma_{m}^{2}}\right) \\
& f_{y}(y)=\sum_{m=0}^{\infty} \frac{e^{-A} A^{m}}{m ! \sqrt{2 \pi \sigma_{m}^{2}}} \exp \left(-\frac{|y|^{2}}{2 \sigma_{m}^{2}}\right) .
\end{aligned}
$$

The cross correlation between the in-phase component $x$ and the quadrature component $\mathrm{y}$ is obtained as $\overline{x y}=\int_{-\infty-\infty}^{\infty} \int_{\infty}^{\infty} x y f(x, y) d x d y=0$. Hence, the in-phase and quadrature components of the class-A impulsive noise are uncorrelated. On the other hand, from (10), (11) and (12), it is clear that the joint pdf of the in-phase and the quadrature components $f_{x, y}(x, y)$ is not equal to the product of the pdf's of each 
component $f_{x}(x) \cdot f_{y}(y)$, i.e. the in-phase and quadrature components of class-A impulsive noise are uncorrelated but dependent.

Assume equal probabilities of signal points and using the joint pdf of the in-phase and quadrature components, the symbol error probability with the proper consideration of the dependence between the in-phase and quadrature components of the noise becomes:

$$
p_{s}(E)=1-\int_{-\infty}^{d / 2} \int_{-\infty}^{d / 2} f_{x, y}(x, y) d x d y
$$

by using (10), the symbol error probability can be expressed as:

$$
p_{s}(E)=1-\sum_{m=0}^{\infty} \frac{A^{m} e^{-A}}{m !}\left(1-\frac{1}{2} \operatorname{erfc}\left(\frac{d}{\sqrt{2} \cdot 2 \sigma_{m}}\right)\right)^{2}
$$

where $d$ is the distance between the two signals. In case of QPSK, it is clear that $d=\sqrt{2 E_{s}}$, where $E_{s}$ is the per-symbol signal energy. Since in QPSK, $E_{s}=2 E_{b}$, where $E_{b}$ is the energy per bit, then, the bit error probability can be expressed as:

$$
p_{b}(E)=1-\sum_{m=0}^{\infty} \frac{A^{m} e^{-A}}{4 \cdot m !}\left(\operatorname{erfc}\left(-\sqrt{\frac{E_{b}}{2 \sigma_{m}^{2}}}\right)\right)^{2}
$$

The mean variance of the class-A impulsive noise $\sigma_{m}^{2}$ can be expressed as $\sigma_{m}^{2}=\sigma_{n}^{2} \sigma_{m}^{\prime 2}$, where $\sigma_{m}^{\prime 2}=\frac{(m / A)+\Gamma}{1+\Gamma}$, then using these relations and using the approximation of the infinite sum, the bit error probability can be expressed as:

$$
p_{b}(E)=1-\max _{m=0,1,2}\left\{\frac{A^{m} e^{-A}}{4 \cdot m !}\left(\operatorname{erfc}\left(-\sqrt{\frac{S N R}{\sigma_{m}^{\prime 2}}}\right)\right)^{2}\right\}
$$

where $S N R=E_{b} / 2 \sigma_{n}^{2}$. Note that, the bit error probability is a function of the signal to noise ratio in addition to the noise parameters $A$ and $\Gamma$. The plot of $p_{b}(E)$ as a function of the SNR and for different values of $A$ is shown in Fig. 3.

\section{Computer Simulations and Results}

In this section, the performance of the developed receiver is evaluated under class-A impulsive noise environment. The performance of the receiver is evaluated in terms of 
bit error probability versus signal to noise ratio (SNR). The parameters used in the simulation are as follows: The transmitted signal is QPSK which represented four hypotheses. The frame length is 2048 samples and the number of the samples per symbol is 6 samples. A class-A impulsive noise is simulated and added to the signal at the input of the receiver.

Analytical and simulation results for the performance of the developed receiver are shown in Fig. 3. The figure is plotted for $\Gamma=1$ and for $A=0.001,0.01,0.1$, and 1 . The figure shows that the simulation results agree with the analytical ones. The figure also shows that the receiver performance deteriorates as the values of the impulsive index $A$ become smaller. This is because as the impulsive index $A$ becomes smaller, the noise impulsiveness becomes stronger, thus causing larger performance degradation. The curve for $A=1$ has the best performance because when $A$ becomes large, the statistical characteristics of the impulsive noise approach those of the Gaussian one.

In Fig. 4, the impulsive index $A$ is assumed to be 1 and the mean power ratio $\Gamma$ of the Gaussian to the impulsive noise varies among the values $0.001,0.1,1$, and 10 . The results show that when $A=1$ and $\Gamma$ becomes larger, the performance of the receiver is improved. This is because the impulsiveness becomes weaker when $\Gamma$ becomes larger. For $\Gamma \geq 10$, the impulsive noise approaches the Gaussian one implying better performance.

The effect of the frequency offset on the performance of the developed receiver is shown in fig. 5. The figure is plotted for $\mathrm{SNR}=3,5$ and $10 \mathrm{~dB}$ and for $A=0.0001,0.01$, 0.1 and 10 . This figure shows that the receiver is able to detect the signal reliably when the frequency offset is small. When the frequency offset increases, the receiver performance degrades rapidly. The figure also shows that there is a range in which the effect of frequency offset can be neglected and the probability of error in this range is small. This range depends on the SNR and the value of impulsive index $A$. It increases as SNR increases.

\section{Conclusions}

An optimum receiver for digitally modulated signals in additive class-A impulsive noise has been presented. The performance of the receiver has been derived theoretically and validated by simulations for a QPSK signal as a case study. It has been shown that the performance of the developed receiver depends on the noise parameters $A$ and $\Gamma$. Increasing the impulsive index $A$, enhances the performance of the receiver. Also, increasing the mean power ratio $\Gamma$ of the Gaussian to the impulsive noise enhances the performance of the receiver. 


\section{REFERENCES}

[1] A. El-Mahdy, "Classification of NFSK Signals over Time Varying Flat Correlated Fading Channels under Class-A Impulsive Noise Environment", IEE proceedings. Communications, vol. 151, No. 6, Dec.2004, pp. 619-626.

[2] S. Kosmopoulos, M. Gouta, and P. Mathiopoulos, "Performance Evaluation of M-ary QPRS Schemes in Sever Impulsive Noise Environments", IEEE Trans. Commun., vol.39, No. 3, March 1991, pp.405-408.

[3] S. Miyamoto, M. Katayama, and N. Morinaga, "Performance Analysis of QAM Systems Under Class-A Impulsive Noise Environment", IEEE Trans. Electomagn. Compat., vol. 37, No. 2, May 1995, pp. 260-167.

[4] J. Haring, and A. Vinck, "Performance Bounds for Optimum and Suboptimum Reception Under Class-A Impulsive Noise", IEEE Trans. Commun., vol. 50, No. 7, July 2002, pp. 1130-1136.

[5] D. Middelton, "Non-Gaussian Noise Models in Signal Processing for Telecommunications: New Methods and Results for Class-A and Class-B Noise Models," IEEE Trans. Inform. Theory, vol. 45, No.4, May. 1999, pp. 11291149 .

[6] S. Zabin and H. Poor, "Parameter Estimation for Middleton Class-A Interference Processes", IEEE Trans. Commun., vol. 37, No. 10, Oct. 1989, pp.1042-1051.

[7] A. D. Whalen, Signal Detection in Non-Gaussian Noise. Dowden \& Culver, Inc., 1988. 


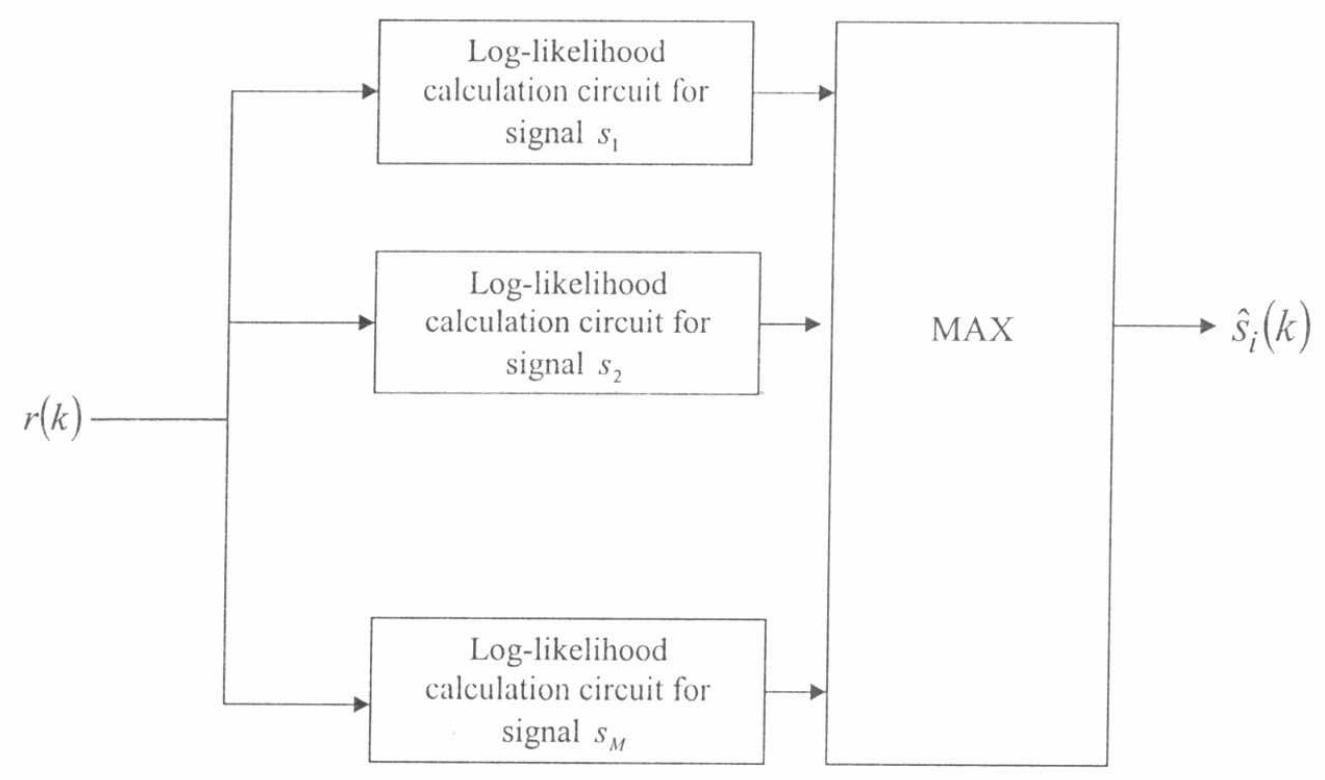

(a)

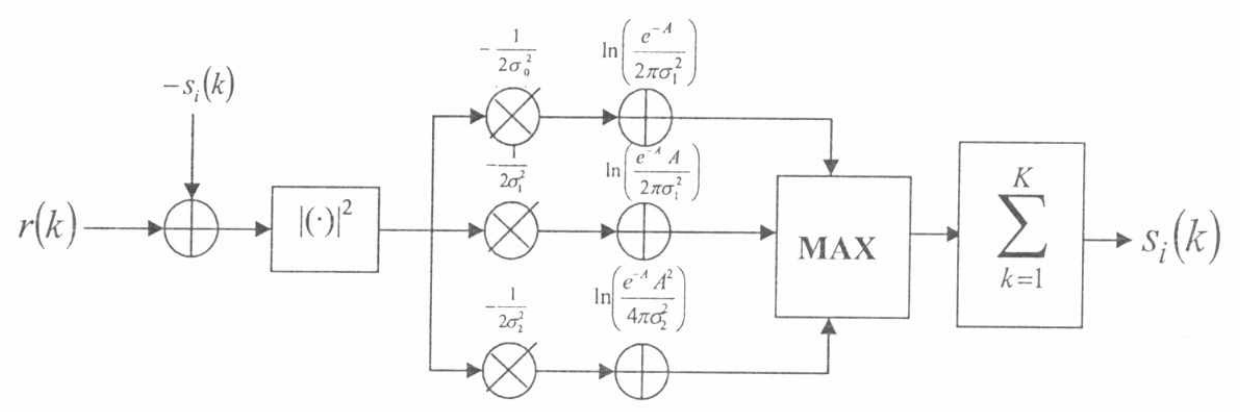

(b)

Fig. 1. Construction of the $M L$ receiver under class-A impulsive noise 
Proceeding of the 11-th ASAT Conference, 17-19 May 2005

Q...Quadrature component

I... In-phase component

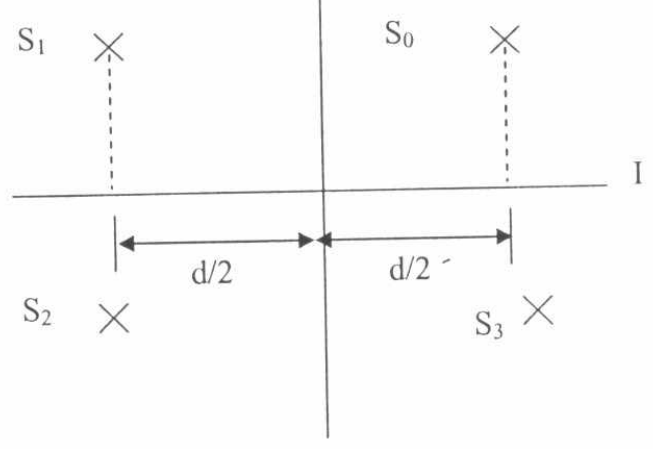

Fig. 2: The signal constellation of QPSK

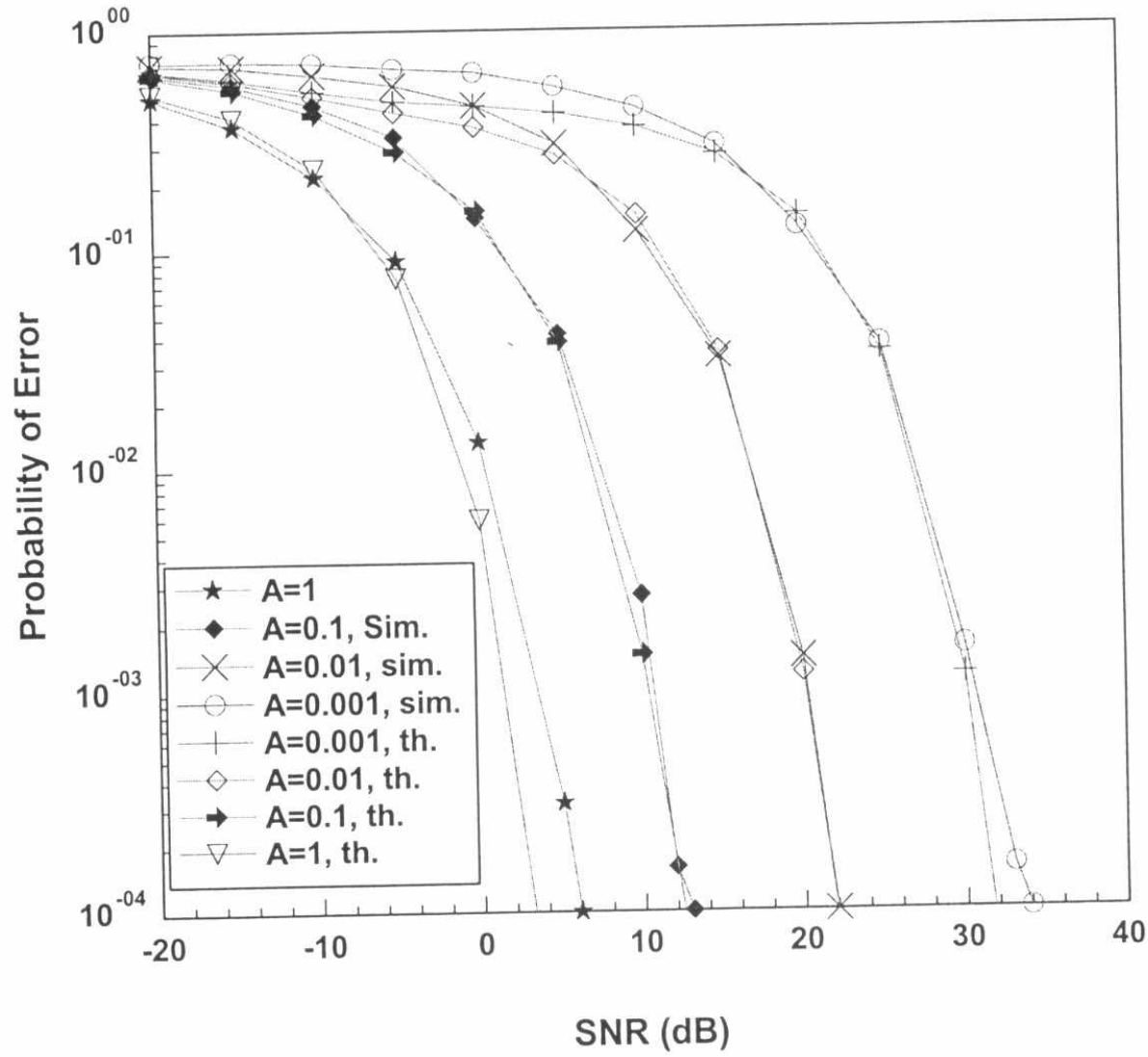

Fig. 3. Theoretical and simulation performance of the receiver for different values of the impulsive index $A$ and for $\Gamma=1$ 


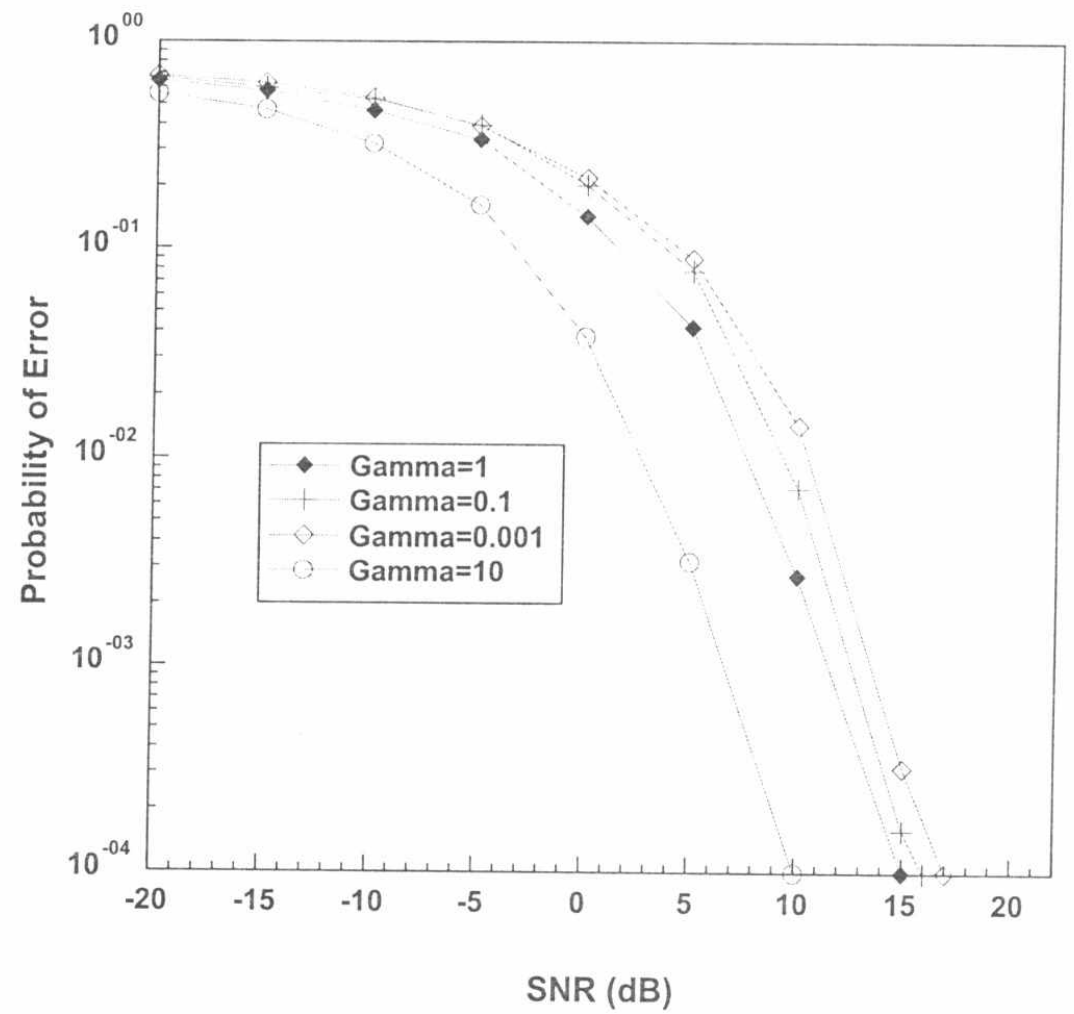

Fig. 4. Probability of error for different values of $\Gamma$ and for $A=1$ 